\title{
Outsourcing Human Drug Compounding
}

National Cancer Institute

\section{Source}

National Cancer Institute. Outsourcing Human Drug Compounding. NCI Thesaurus.

Code C82434.

Compounding drug dosage forms for human consumption under a contract by other sources than the facilities that use the pharmaceutics. 